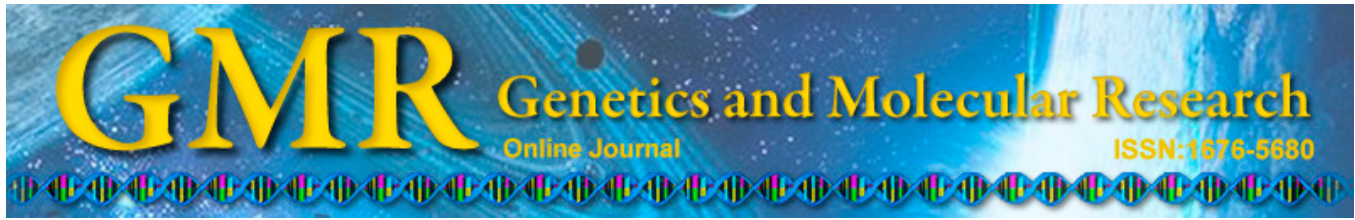

\title{
Multivariate analysis of molecular markers in peripheral blood associated with recurrence and metastasis of hepatocellular carcinoma
}

\author{
X.J. Liu ${ }^{1,2}$, B. Wang ${ }^{1,3}$, W.G. Jiang ${ }^{3}$, Y.J. Li ${ }^{3}$, J.B. Liu' ${ }^{2}$ and M. Zhang ${ }^{2}$ \\ ${ }^{1}$ Shandong University School of Medicine, Jinan, Shandong, China \\ ${ }^{2}$ Binzhou Medical College Hospital, Binzhou, Shandong, China \\ ${ }^{3}$ Binzhou Medical College, Yantai, Shandong, China
}

Corresponding author: B. Wang

E-mail: BinWangdoc@163.com

Genet. Mol. Res. 14 (1): 1502-1507 (2015)

Received April 4, 2014

Accepted October 7, 2014

Published February 20, 2015

DOI http://dx.doi.org/10.4238/2015.February.20.5

\begin{abstract}
Invasion, metastasis, and recurrence are the most common causes of death in patients with hepatocellular carcinoma (HCC) and are therefore critical factors for both therapy and prognosis. Current methods for diagnosis of HCC rely mainly on serological markers such as alpha-fetoprotein and liver enzymes, together with physical assessment and imaging techniques. The availability of more accurate serum markers may facilitate screening and early diagnosis, which will improve prognosis. This retrospective cohort analysis included 50 consecutive patients with cirrhosis and single or multifocal HCC and 40 control subjects with no liver disease or risk factors for viral hepatitis. Expression of epidermal growth factor-like domain 7 (EGFL7), osteopontin (OPN), and prostaglandin E2 (PGE2) were
\end{abstract}


detected using an enzyme-linked immunosorbent assay. The mean serum levels of EGFL7, OPN, and PGE2 in the HCC group were 132.11 $\mathrm{pg} / \mathrm{mL}, 11.77 \mathrm{ng} / \mathrm{mL}$, and $179.37 \mathrm{pg} / \mathrm{mL}$, respectively, which were all significantly higher than the levels in the control group $(23.03 \mathrm{pg} / \mathrm{mL}$, $2.31 \mathrm{ng} / \mathrm{mL}$, and $47.36 \mathrm{pg} / \mathrm{mL}$, respectively; $\mathrm{P}<0.001$ ). Serum levels of EGFL7, OPN, and PGE2 levels may thus be useful for screening and surveillance of HCC among high-risk populations, and have the potential to improve prognosis of these patients.

Key words: Hepatocellular carcinoma; EGFL7; OPN; PGE2; Marker; Multivariate analysis

\section{INTRODUCTION}

Hepatocellular carcinoma (HCC) is the fifth most common cancer and the third most common cause of cancer-related death worldwide (Yeo et al., 2007; Du and Cao, 2012). Since the 1990s, the incidence of $\mathrm{HCC}$ in China has remained stable, with an annual mortality rate of 20.4 per 100,000 . Over $90 \%$ of liver cancers are HCC, of which $75-80 \%$ are related to chronic viral infection and $80-90 \%$ develop from, or are combined with, liver cirrhosis (Bartolomeo et al., 2011). Despite improvements in surveillance, diagnosis, and clinical treatment strategies in recent years, the prognosis of HCC remains poor. In fact, invasion, metastasis, and recurrence are the most common causes of death among HCC patients (Raskopf et al., 2009). Accordingly, identification of serum markers that could facilitate early detection and surveillance of HCC in high-risk patients could have a significant influence on the mortality rate associated with this cancer.

Unfortunately, the most widely used serum markers for HCC cannot detect all types of HCC (Sterling et al., 2012). For example, although alpha-fetoprotein (AFP) is a widely used marker (Bertino et al., 2012; Masuzaki et al., 2012), because HCC is negative for AFP in most cases, AFP does not enable early differential diagnosis of HCC and has shown consistent results for the detection recurrence/metastasis. Consequently, it is important to identify new methods for the early detection and surveillance of HCC (Lu et al., 2005; Zhou et al., 2006).

The available evidence indicates that mechanisms underlying both the pathogenesis and metastasis of HCC are complicated, and involve a combination of genetic and epigenetic aberrations. Nevertheless, recent data on the development, invasion, and metastasis of HCC have suggested the use of three novel markers for the detection and surveillance of HCC. These include epidermal growth factor-like domain 7 (EGFL7), osteopontin (OPN), and prostaglandin E2 (PGE2). EGFL7 has been found to act as a chemoattractant for cell migration and may regulate collective migration of endothelial cells, indicating an important role in cell motility (Wu et al., 2009; Fan et al., 2013). OPN is a multifunctional protein involved in pathological conditions including inflammation, immunity, angiogenesis, fibrosis, and cancer progression in various tissues. In patients with HCC, the plasma OPN level has been described as a prognostic factor after liver resection or transplantation (Xue et al., 2013; Nagoshi, 2014). PGE2 has been implicated in cell growth and metastasis in many types of cancers. Bai et al. (2009) found that treatment with PGE2 significantly increased cell adhesion, migration, and invasion of HCC cells. Therefore, the present study was designed to 
determine whether these markers may be useful to improve the sensitivity and specificity of AFP.

\section{MATERIAL AND METHODS}

\section{Subjects}

This retrospective study included 50 consecutive patients with cirrhosis and single or multifocal HCC. Patients had been treated with intra-arterial therapy and evaluated with at least two dynamic imaging studies (dynamic computed tomography and angiography) at a single center between July 2008 and June 2011. Patients who underwent at least one imaging examination (16-slice helical computed tomography or triphasic contrast-enhanced magnetic resonance imaging) before and after treatment were included. Diagnosis of HCC was based on radiological findings, AFP levels, and biopsy according to the Barcelona criteria (Bruix et al., 2001). Impairment of liver function was estimated using routine biochemical parameters reflecting liver function. The control subjects enrolled were hospital staff with no liver disease or risk factors for viral hepatitis and normal physiological conditions.

Blood was assayed for the following markers: aspartate aminotransaminase, alanine aminotransaminase, AFP, gamma-glutamyl transpeptidase, alkaline phosphatase, carbohydrate antigen 19-9, carbohydrate antigen 125 , and carcinoembryonic antigen. The characteristics of the $50 \mathrm{HCC}$ patients and 40 control subjects are reported in Table 1. This study was conducted in accordance with the Declaration of Helsinki, and was approved by the ethics committee of Binzhou Medical College. Written informed consent was obtained from all participants.

\section{Enzyme-linked immunosorbent assay (ELISA) analysis}

Blood samples $(4 \mathrm{~mL})$ obtained before initiation of treatment for HCC were drawn from each subject and centrifuged, and the serum was stored at $-80^{\circ} \mathrm{C}$ until assayed. Serum levels of EGFL7, OPN, and PGE2 were measured using an ELISA kit (Rapid Bio, CA, USA). ELISA plates were pre-coated with monoclonal antibodies against EGFL7, OPN, or PGE2. A second horseradish peroxidase -labeled antibody to EGFL7, OPN, or PGE2 enabled quantification of serum markers following reaction with 3,3',5,5'-tetramethylbenzidine. Absorbance was measured at $450 \mathrm{~nm}$ in a microplate reader (Bio-Rad 680). Standard curves were generated for each reaction.

\section{Statistical analysis}

An unpaired Student $t$-test was used to compare averages between groups for normally distributed data, while the Kruskal-Wallis test was used to assess differences between groups when data failed tests of normality or equal distribution. A P value $<0.05$ was considered to be statistically significant. In addition to age, gender, and variables that achieved statistical significance with univariate analysis, data were subsequently assessed by multivariate analysis using a Cox proportional hazards model. Analyses were performed using the StatView statistics package (SAS Institute, Cary, NC, USA). 


\section{RESULTS}

\section{Patient characteristics}

Demographic data and serum marker levels assessed in the 50 HCC patients and 40 control subjects are summarized in Table 1. While there were no differences between HCC patients and control subjects in age and gender, there were significant differences in the serum levels of all markers between groups.

Table 1. Characteristics of patients with hepatocellular carcinoma and control subjects

\begin{tabular}{lccc}
\hline Variable $^{1}$ & Patients with HCC $(\mathrm{N}=50)$ & Controls $(\mathrm{N}=40)$ & P \\
\hline Age & $53.74 \pm 10.51$ & $45.30 \pm 10.24$ & $>0.05$ \\
Gender (M/F) (N) & $37 / 13$ & $28 / 12$ & $>0.05$ \\
ALT (U/L) & $111.68(17.30-578.70)$ & $19.90(8.40-42.30)$ & $<0.0001$ \\
AST (U/L) & $217.86(19.00-1246.10)$ & $24.03(15.50-41.60)$ & $<0.0001$ \\
GGT (U/L) & $158.84(35.50-611.40)$ & $21.30(7.90-49.00)$ & $<0.0001$ \\
ALP (U/L) & $199.77(54.40-1179.90)$ & $100.64(52.40-150.90)$ & $<0.0001$ \\
AFP (U/L) & $1106.70(248.00-1210.00)$ & $8.04(2.19-12.31)$ & $<0.0001$ \\
CEA (U/L) & $4.24(1.20-19.34)$ & $2.63(1.38-4.64)$ & $<0.05$ \\
CA-19-9 (U/L) & $131.33(12.44-2244.00)$ & $22.45(11.63-33.75)$ & $<0.0001$ \\
CA-125 (U/L) & $39.97(3.80-263.50)$ & $17.19(5.78-28.26)$ & $<0.0001$ \\
\hline
\end{tabular}

${ }^{1}$ Data are reported as median values (range minimum-maximum), or means \pm SD if normally distributed. ALT, alanine aminotransaminase; AST, aspartate aminotransaminase; GGT, gamma-glutamyl transpeptidase; ALP, alkaline phosphatase; AFP, alpha fetoprotein; CEA, carcinoembryonic antigen; CA-19-9, carbohydrate antigen 19-9; CA-125, carbohydrate antigen 125.

\section{Measurement of EGFL7, OPN, and PGE2 levels in the serum of HCC and healthy control subjects}

Sandwich ELISA was performed to measure the levels of EGFL7, OPN, and PGE2, and the quality of the assay was controlled with a five-point calibration curve. The mean serum levels of EGFL7, OPN, and PGE2 in the HCC group were $132.11 \mathrm{pg} / \mathrm{mL}, 11.77 \mathrm{ng} / \mathrm{mL}$, and $179.37 \mathrm{pg} / \mathrm{mL}$, respectively, which were significantly higher than those in the control group (23.03 pg/mL, $2.31 \mathrm{ng} / \mathrm{mL}$, and $47.36 \mathrm{pg} / \mathrm{mL}$, respectively, $\mathrm{P}<0.001$; Figure 1).
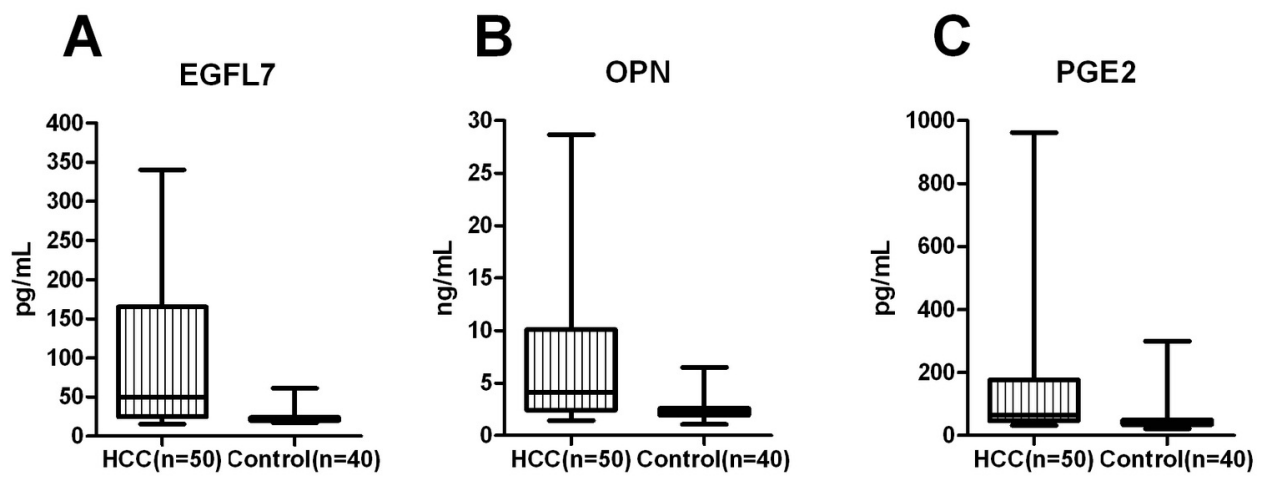

Figure 1. Expressions of EGFL7, OPN, and PGE2 in two groups. Three black lines from top to bottom indicated the maximum, median, and minimum. $\mathrm{P}<0.01$ for EGFL7, OPN, and PGE2 comparing control vs HCC group. 


\section{DISCUSSION}

Several new candidate markers for HCC that have the potential to increase the sensitivity and specificity of diagnosis have been identified in the serum. Current methods for diagnosis of HCC mainly rely on serological markers such as AFP (Baig et al., 2009) and liver enzymes, together with physical assessments and imaging techniques (e.g. ultrasonography or computed tomography) (Sogawa et al., 2012). While these methods have good overall sensitivity, the high mortality rates associated with HCC suggest that better serum markers are needed for both screening and early diagnosis.

EGFL 7 has been shown to play a role in angiogenesis in HCC, which is a highly vascularized tumor. Thus, we hypothesized that EGFL7 might be a novel serological diagnostic marker for HCC (Nichol et al., 2010; Nichol and Stuhlmann, 2012). Quality control of sandwich ELISA for serum EGFL7 enabled reliable detection of highly significant differences in the level of this marker between HCC patients and control subjects. EGFL7 is expressed at high levels during embryonic development, and downregulated in almost all mature tissues except lung, heart, and kidney; it is strongly upregulated in many actively proliferating tissues, including tumors (Nichol and Stuhlmann, 2012). This unique expression pattern, which is similar to that of the embryonic proteins AFP and carcinoembryonic antigen, indicates the potential of EGFL7 as a novel tumor biomarker. The expression of OPN is closely related to that of vascular endothelial growth factor, and there is evidence that it may play a role in tumor angiogenesis (Abu et al., 2011; Zhou et al., 2013), suggesting that OPN may also be a marker for early detection of HCC. A recent study demonstrated that higher expression of OPN is significantly associated with advanced histological grade, advanced pathological stage, and poorer survival in HCC (Tsai et al., 2012). We hypothesized that OPN may be a marker for metastasis of HCC based on evidence that levels of OPN increase significantly in subjects with recurrent and metastasized HCC. Finally, PGE2 has been reported to play an essential role in promoting the growth of human HCC cells through the Wnt/- $\beta$ catenin pathway (Lim et al., 2009), thus substantiating our prediction that serum levels of PGE2 may also be a novel marker for HCC.

Consistent with previous findings, the present data suggest that the levels of each of these three markers are significantly elevated in HCC patients relative to the controls. However, despite the high sensitivity of ELISA, none of these novel potential markers appeared to have a sensitivity equal to that of more standard markers such as AFP. Further studies are needed to determine whether these novel markers have increased sensitivity in early detection, recurrence, or metastasis of $\mathrm{HCC}$.

\section{REFERENCES}

Abu El Makarem MA, Abdel-Aleem A, Ali A, Saber R, et al. (2011). Diagnostic significance of plasma osteopontin in hepatitis C virus-related hepatocellular carcinoma. Ann. Hepatol. 10: 296-305.

Bai XM, Zhang W, Liu NB, Jiang H, et al. (2009). Focal adhesion kinase: important to prostaglandin E2-mediated adhesion, migration and invasion in hepatocellular carcinoma cells. Oncol. Rep. 21: 129-136.

Baig JA, Alam JM, Mahmood SR, Baig M, et al. (2009). Hepatocellular carcinoma (HCC) and diagnostic significance of A-fetoprotein (AFP). J. Ayub Med. Coll. Abbottabad 21: 72-75.

Bartolomeo N, Trerotoli P and Serio G. (2011). Progression of liver cirrhosis to HCC: an application of hidden Markov model. BMC Med. Res. Methodol. 11: 38.

Bertino G, Ardiri A, Malaguarnera M, Malaguarnera G, et al. (2012). Hepatocellualar carcinoma serum markers. Semin. 
Oncol. 39: 410-433.

Bruix J, Sherman M, Llovet JM, Beaugrand M, et al. (2001). Clinical management of hepatocellular carcinoma. Conclusions of the Barcelona-2000 EASL Conference. European Association for the Study of the Liver. J. Hepatol. 35: 421-430.

Du Y and Cao GW (2012). Challenges of incorporating gene expression data to predict HCC prognosis in the age of systems biology. World J. Gastroenterol. 18, 3941-4.

Fan C, Yang LY, Wu F, Tao YM, et al. (2013). The expression of EGFL7 in human normal tissues and epithelial tumors. Int. J. Biol. Marker. 28: 71-83.

Lim K, Han C, Dai Y, Shen M, et al. (2009). Omega-3 polyunsaturated fatty acids inhibit hepatocellular carcinoma cell growth through blocking beta-catenin and cyclooxygenase-2. Mol. Cancer Ther. 8: 3046-3055.

Lu ZL, Luo DZ and Wen JM (2005). Expression and significance of tumor-related genes in HCC. World J. Gastroenterol. 11: 3850-3854.

Masuzaki R, Karp SJ and Omata M (2012). New serum markers of hepatocellular carcinoma. Semin. Oncol. 39: 434-439. Nagoshi S (2014). Osteopontin: Versatile modulator of liver diseases. Hepatol. Res. 44: 22-30.

Nichol D and Stuhlmann H (2012). EGFL7: a unique angiogenic signaling factor in vascular development and disease. Blood 119: 1345-1352.

Nichol D, Shawber C, Fitch MJ, Bambino K, et al. (2010). Impaired angiogenesis and altered Notch signaling in mice overexpressing endothelial EGFL7. Blood 116: 6133-6143.

Raskopf E, Gerceker S, Vogt A, Standop J, et al. (2009). Plasminogen fragment K1-3 inhibits expression of adhesion molecules and experimental HCC recurrence in the liver. Int. J. Colorectal Dis. 24: 837-844.

Sogawa C, Tsuji AB, Yoshida C, Inubushi M, et al. (2012). Novel human monoclonal antibody against epidermal growth factor receptor as an imaging probe for hepatocellular carcinoma. Nucl. Med. Commun. 33: 719-725.

Sterling RK, Wright EC, Morgan TR, Seeff LB, et al. (2012). Frequency of elevated hepatocellular carcinoma (HCC) biomarkers in patients with advanced hepatitis C. Am. J. Gastroenterol. 107: 64-74.

Tsai WC, Lee HS, Jin JS, Gao HW, et al. (2012). Association between osteopontin and EGFR expression with clinicopathological parameters in hepatocellular carcinoma. Chin. J. Physiol. 55: 412-420.

Wu F, Yang LY, Li YF, Ou DP, et al. (2009). Novel role for epidermal growth factor-like domain 7 in metastasis of human hepatocellular carcinoma. Hepatology 50: 1839-1850.

Xue TC, Chen RX, Ren ZG, Zou JH, et al. (2013). Transmembrane receptor CXCR7 increases the risk of extrahepatic metastasis of relatively well-differentiated hepatocellular carcinoma through upregulation of osteopontin. Oncol. Rep. 30: 105-110.

Yeo W, Mo FK, Chan SL, Leung NW, et al. (2007). Hepatitis B viral load predicts survival of HCC patients undergoing systemic chemotherapy. Hepatology 45: 1382-1389.

Zhou C, Zhou HJ, Zhang XF, Lou LL, et al. (2013). Postoperative serum osteopontin level is a novel monitor for treatment response and tumor recurrence after resection of hepatitis B-related hepatocellular carcinoma. Ann. Surg. Oncol. 20: 929-937.

Zhou SF, Xie XX, Bin YH, Lan L, et al. (2006). Identification of HCC-22-5 tumor-associated antigen and antibody response in patients. Clin. Chim. Acta 366: 274-280. 